\title{
L-arginine supplementation attenuates capillary regression without increasing integrated succinate dehydrogenase activity and VEGF expression in skeletal muscle during hindlimb unloading
}

\author{
Kensaku Uchida ${ }^{1,2}$, Minoru Tanaka ${ }^{1,3}$, Hiroyo Kondo ${ }^{4}$, Akihiko Ishihara ${ }^{5}$ and Hidemi Fujino ${ }^{1}$ \\ ${ }^{1}$ Department of Rehabilitation Science, Kobe University, Graduate School of Health Sciences, 7-10-2 Tomogaoka, \\ Suma-ku, Kobe 654-0142, Japan \\ ${ }^{2}$ Department of Rehabilitation Medicine, Nishinomiya Kyoritsu Neurosurgical Hospital, 11-1 Imazu-Yamanaka-cho, \\ Nishinomiya 663-8211, Japan \\ ${ }^{3}$ Department of Physical Therapy, Osaka Yukioka College of Health Science, 1-1-41, Sojiji, Ibaraki 567-0801, Osaka, Japan \\ ${ }^{4}$ Department of Food Science and Nutrition, Nagoya Women's University, 3-40 Shioji-cho, Mizuho-ku, Nagoya 467-0003, \\ Japan \\ ${ }^{5}$ Laboratory of Cell Biology and Life Science, Graduate School of Human and Environmental Studies, Kyoto University, \\ Yoshida-Nihonmatsu-cho, Sakyo-ku, Kyoto 606-8316, Japan
}

\begin{abstract}
Decreased capillary number is observed in atrophied muscle. The change in capillary number is regulated by angiogenic factors. L-arginine enhances expression of endothelial nitric oxide synthase (eNOS), angiogenic factor, in skeletal muscle. Therefore, the aim of this study was to evaluate the effects of L-arginine supplementation on capillary regression during hindlimb unloading. Twenty-four male Wistar rats were divided into four treatment groups: (1) control, (2) L-arginine supplementation, (3) hindlimb unloading, and (4) hindlimb unloading with L-arginine supplementation. Hindlimb unloading resulted in a decrease of capillary-to-muscle fibre (C/F) ratio, eNOS expression, and integrated succinate dehydrogenase (SDH) activity. L-arginine supplementation attenuated the decrease in both eNOS expression and C/F ratio, although it did not increase integrated SDH activity in skeletal muscle. These results indicate that $\mathrm{L}$-arginine supplementation is effective for maintaining capillary number in atrophied muscle, and that elevation of eNOS expression may be one mechanism associated with these responses.
\end{abstract}

Key words: L-arginine — Muscle atrophy — Capillary regression — Angiogenic factor

Abbreviations: Arg, arginine; C/F ratio, capillary-to-muscle fibre ratio; CON, control; eNOS, endothelial nitric oxide synthase; FCSA, fibre cross-sectional area; HU, hindlimbs unloading; NO, nitric oxide; PBST, phosphate-buffered saline with Tween-20; SDH, succinate dehydrogenase; SDS, sodium dodecyl sulphate; VEGF, vascular endothelial growth factor.

\section{Introduction}

Muscle atrophy results from a variety of conditions, such as inactivity (Adrian et al. 1988), cast immobilization (Booth 1982; Pachter and Eberstein 1984), denervation (Dow et al.

Correspondence to: Hidemi Fujino, Department of Rehabilitation Science, Kobe University, Graduate School of Health Sciences, 7-10-2 Tomogaoka, Suma-ku, Kobe 654-0142, Japan

E-mail: fujino@phoenix.kobe-u.ac.jp
2004; Russo et al. 2007), and spinal-cord injury (Roy et al. 2002; Kim et al. 2008). In addition, inactivity-induced atrophy occurs faster in antigravity muscle, such as slow-twitch muscle, than in fast-twitch muscle due to each muscle's characteristics (Sandonà et al. 2012). It has been well established that muscle mass and fibre cross-sectional area (FCSA) are decreased in atrophied muscle (Desplanches et al. 1987). Capillary number and mitochondrial oxidative enzyme activity are also decreased in atrophied muscle (Desplanches et al. 1987; Fujino et al. 2005; Roudier et al. 2010). Regressed 
capillary has been observed as an adaptive change to decreased oxidative demand in atrophied muscle. In addition, capillary regression induces decreasing endurance capacity in skeletal muscle (Tadaishi et al. 2011) and results in physical inactivity. Therefore, it is necessary to prevent capillary regression under inactivity conditions.

The alteration of capillary number is regulated by angiogenesis-related factors, e.g. vascular endothelial growth factor (VEGF) and nitric oxide (NO) (Hoeben et al. 2004; Milkiewicz et al. 2005; Egginton. 2011; Forstermann and Sessa 2012). Several studies have suggested that the VEGF/endothelial NO synthase (eNOS)/NO pathway is one of the major pathways for regulating angiogenesis (Ziche et al. 1997; Llevadot and Asahara 2002; Kimura and Esumi 2003). VEGF, which works as the initiator in the pathway, increases the eNOS expression level and induces angiogenesis by regulating the eNOS/ NO step of the pathway. In addition, it has been shown that inactivity conditions induce a decrease of VEGF expression (Kaneguchi et al. 2014). Therefore, it is important to maintain VEGF expression to prevent capillary regression.

$\mathrm{NO}$ is produced from L-arginine by the catalytic action of eNOS with several coenzymes (El-Hattab et al. 2012). In addition, eNOS is essential for migration during angiogenesis (Murohara et al. 1999). However, the levels of eNOS expression (Schrage et al. 2000) and NO concentration (Lomonosova et al. 2011), as well as of VEGF expression, are decreased under inactivity conditions. Meanwhile, L-arginine supplementation can enhance the level of eNOS expression (Javanmard et al. 2009). Therefore, L-arginine supplementation seems to be a simple and effective intervention to enhance the level of eNOS expression. L-arginine supplementation could increase capillary number in skeletal muscle through the increased eNOS expression and activate the VEGF/eNOS/NO pathway under physical inactivity conditions. In addition, L-arginine supplementation attenuated the decrease in NO concentration in the soleus muscles during hindlimb unloading (Lomonosova et al. 2011). Therefore, we hypothesized that L-arginine supplementation would attenuate the regression of capillary number in skeletal muscle by activating the VEGF/eNOS/NO pathway during hindlimb unloading. If the hypothesis is verified, L-arginine supplementation would be a helpful strategy for the clinical rehabilitation of disuse-syndrome patients, because of the link to exercise-endurance capacity. The aim of the present study was to evaluate the effect of L-arginine supplementation on capillary regression in hindlimb unloading.

\section{Materials and Methods}

\section{Experimental groups and conditions}

Twenty-four male Wistar rats (8 weeks old, body weight: 180-200 g) were purchased from Japan SLC (Hamamatsu,
Japan). After a 1-week familiarization period, the rats were randomly divided into the following four groups: (1) control (CON, $n=6$ ), (2) control with L-arginine supplementation (CON+Arg, $n=6)$, (3) hindlimb unloading (HU, $n=6)$, and (4) hindlimb unloading with L-arginine supplementation (HU+Arg, $n=6)$. All rats were housed in an environmentally controlled room at $22 \pm 2{ }^{\circ} \mathrm{C}$ with a 12-h light/dark cycle, and given food and drinking water ad libitum. This study was approved by the Institutional Animal Care and Use Committee (Protocol No. P130903-R1), and was performed according to the Kobe University Animal Experimentation Regulations. All experiments were conducted in accordance with the National Institute of Health Guide for the Care and Use of Laboratory Animals (National Research Council, USA, 1996).

\section{Hindlimb unloading}

Hindlimb unloading was induced by suspending the rats by their tails for 7 days, according to the method described by Morey (Morey et al. 1979). Briefly, each rat in the HU and $\mathrm{HU}+\mathrm{Arg}$ groups was fitted with a tail harness and suspended by a string, just high enough to prevent the hindlimbs from bearing weight on the floor or sides of the cage. The forelimbs were allowed to maintain contact with the floor of the cage.

\section{L-arginine supplementation}

L-arginine supplementation was started at 9 weeks of age, and administered during the hindlimb unloading period. The rats in the $\mathrm{CON}+\mathrm{Arg}$ and $\mathrm{HU}+\mathrm{Arg}$ groups were orally administered L-arginine (1,500 $\mathrm{mg} / \mathrm{kg}$ body weight/day; 03321-65, Nacalai Tesque, Kyoto, Japan) in solution via a sonde, twice a day. The L-arginine solution was prepared by dissolving $27 \%$ L-arginine (w/v) in distilled water and adjusting the $\mathrm{pH}$ to 7.4 . The rats in the $\mathrm{CON}$ and $\mathrm{HU}$ groups were administered the same volume (approximately $0.6 \mathrm{ml}$ ) of distilled water.

\section{Histological analyses}

After 7 days of intervention, all rats were weighed and deeply anaesthetized with sodium pentobarbital $(50 \mathrm{mg} /$ $\mathrm{kg}$, intraperitoneal injection). Under anaesthetized conditions, both soleus muscles were removed. The muscles were weighed, rapidly frozen in dry-ice-cooled acetone, mounted on cork, and stored at $-80^{\circ} \mathrm{C}$ until use in histological and biochemical analyses. A block from the mid-region of the muscle was mounted on cork and tissue cross-sections of $12-\mu \mathrm{m}$ thickness were obtained by using a cryostat (CM1510 S, Leica Microsystems, Mannheim, Germany) at $-25^{\circ} \mathrm{C}$. The sections were stained for alkaline phosphatase (AP) to 
visualize capillaries, according to the method described by Hansen-Smith et al. (1992). The sections were then incubated in $0.1 \%$ nitro blue tetrazolium, $0.1 \%$ 5-bromo-4-chloro-3indolyl phosphate $p$-toluidine salt, and $0.01 \mathrm{M}$ magnesium sulphate in $0.2 \mathrm{M}$ borate buffer for $60 \mathrm{~min}$ at $37^{\circ} \mathrm{C}$, and fixed with $4 \%$ paraformaldehyde. The capillary-to-muscle fibre $(\mathrm{C} / \mathrm{F})$ ratio was calculated from the stained sections of at least 300 individual capillaries using microscopic images of the AP staining. The activity of succinate dehydrogenase (SDH) was also measured in the sections, according to the method described by Nachlas et al. (1957). In brief, the sections were incubated at $37^{\circ} \mathrm{C}$ in $0.2 \mathrm{M}$ sodium succinate and $0.05 \%$ nitro blue tetrazolium in $0.2 \mathrm{M}$ phosphate buffer, $\mathrm{pH} 7.6$, for $1 \mathrm{~h}$. The SDH-stained sections were used to calculate FCSA and the mean value of SDH activity. Densitometry data on SDH activity were analysed by using the Image J software program (NIH, Bethesda, MD, USA). The FCSA was also measured in the SDH-stained sections and the results were analysed by using the same software program. At least 150 muscle fibres per individual were assessed for SDH activity and FCSA. The integrated SDH activities were calculated by a previously described method (Chalmers et al. 1991; Bekedam et al. 2003).

\section{Western blot analysis}

The expression of VEGF and eNOS in the soleus muscles were quantified by Western blotting. A portion $(\sim 20 \mathrm{mg})$ of each soleus muscle was homogenized in ice-cold homogenizing buffer (50 mM Tris-HCl, $120 \mathrm{mM} \mathrm{NaCl}, 1 \mathrm{mM}$ EDTA, 1\% NP-40, pH 7.4). The homogenates were then centrifuged for $10 \mathrm{~min}\left(15,000 \times g, 4^{\circ} \mathrm{C}\right)$. Total protein concentration was determined with a protein determination kit (Bio-Rad, Hercules, CA, USA). The homogenates were solubilized in sample loading buffer, containing $50 \mathrm{mM}$ Tris- $\mathrm{HCl}, \mathrm{pH}$ 6.8, 2\% sodium dodecyl sulphate (SDS), $10 \%$ glycerol, $5 \% \beta$-mercaptoethanol, and $0.005 \%$ bromophenol blue, and heated for $10 \mathrm{~min}$ at $80^{\circ} \mathrm{C}$. Sample proteins $(30-40 \mu \mathrm{g})$ were separated by $7.5 \%$ (for eNOS) or $12.5 \%$ (for VEGF) SDS-polyacrylamide gel electrophoresis, and transferred to polyvinylidene fluoride membranes. The membranes were blocked for $1 \mathrm{~h}$ in $5 \%$ skimmed milk in phosphate-buffered saline with Tween-20 (PBST), and incubated with a primary antibody against eNOS (1:100; sc-654, Santa Cruz Biotechnology, Dallas, TX, USA) or VEGF (1:200; sc-7269, Santa Cruz Biotechnology) in PBST overnight at $4^{\circ} \mathrm{C}$. The membranes were then incubated for $60 \mathrm{~min}$ at room temperature with anti-mouse or anti-rabbit IgG conjugated to horseradish peroxidase (GE Healthcare, Waukesha, WI, USA). The membrane was developed using a chemiluminescent reagent (ECL plus, GE Healthcare) and analyzed with an image reader (LAS-1000, Fujifilm, Tokyo, Japan). A $\beta$-actin antibody (1:200; sc-47778, Santa Cruz Biotechnology) was used as an internal control.

\section{Statistical analysis}

All data are presented as the mean \pm SEM. Differences were assessed by two-way analysis of variance (ANOVA). The Tukey-Kramer post-hoc test was conducted if the two-way ANOVA indicated a significant difference. For all tests, values of $p<0.05$ were considered statistically significant.

\section{Results}

Body weight, soleus muscle wet weight, relative muscle-to-body weight, and fibre cross-sectional area

Table 1 shows the results for mean body weight, soleus muscle wet weight, relative muscle-to-body weight and FCSA. After 7 days of unloading, the body weights of the HU and $\mathrm{HU}+\mathrm{Arg}$ groups were significantly lower than those of the $\mathrm{CON}$ and $\mathrm{CON}+\mathrm{Arg}$ groups. For the soleus muscles, muscle wet weights, relative muscle-to-body weights and FCSA were significantly lower in the $\mathrm{HU}$ and $\mathrm{HU}+\mathrm{Arg}$ groups than in the $\mathrm{CON}$ and $\mathrm{CON}+\mathrm{Arg}$ groups. There was no significant difference in the relative muscle-to-body weight of the soleus muscles between the $\mathrm{CON}$ and $\mathrm{CON}+\mathrm{Arg}$ groups, or between the $\mathrm{HU}$ and $\mathrm{HU}+\mathrm{Arg}$ groups.

Table 1. Body weight, soleus muscle wet weight, relative muscle-to-body weight, and fibre cross-sectional area (FCSA)

\begin{tabular}{lcccc}
\hline Group & $\begin{array}{c}\text { Body weight } \\
(\mathrm{g})\end{array}$ & $\begin{array}{c}\text { Muscle wet weight } \\
(\mathrm{mg})\end{array}$ & $\begin{array}{c}\text { Relative muscle weight } \\
\left(\times 10^{-2} \mathrm{mg} / \mathrm{g}\right)\end{array}$ & $\begin{array}{c}\text { FCSA } \\
\left(\mu \mathrm{m}^{2}\right)\end{array}$ \\
\hline CON & $230 \pm 2$ & $92 \pm 2$ & $39.8 \pm 0.8$ & $1965 \pm 117$ \\
CON+Arg & $232 \pm 3$ & $91 \pm 2$ & $39.5 \pm 0.8$ & $1887 \pm 75$ \\
HU & $212 \pm 2^{*, \dagger}$ & $62 \pm 1^{*, \dagger}$ & $29.4 \pm 0.6^{*, \dagger}$ & $1060 \pm 67^{*, \dagger}$ \\
HU+Arg & $209 \pm 3^{*, \dagger}$ & $63 \pm 1^{*, \dagger}$ & $30.4 \pm 0.4^{*, \dagger}$ & $1098 \pm 75^{*, \dagger}$ \\
\hline
\end{tabular}

Values are the mean \pm SEM. $n=6$. CON, control (no treatment); CON+Arg, control with L-arginine supplementation; $\mathrm{HU}$, hindlimb unloading (for 7 days); $\mathrm{HU}+\mathrm{Arg}$, hindlimb unloading with L-arginine supplementation. ${ }^{*}$ and ${ }^{\dagger}$ indicate significant differences from $\mathrm{CON}$ and $\mathrm{CON}+\mathrm{Arg}$, respectively, at $p<0.05$. 

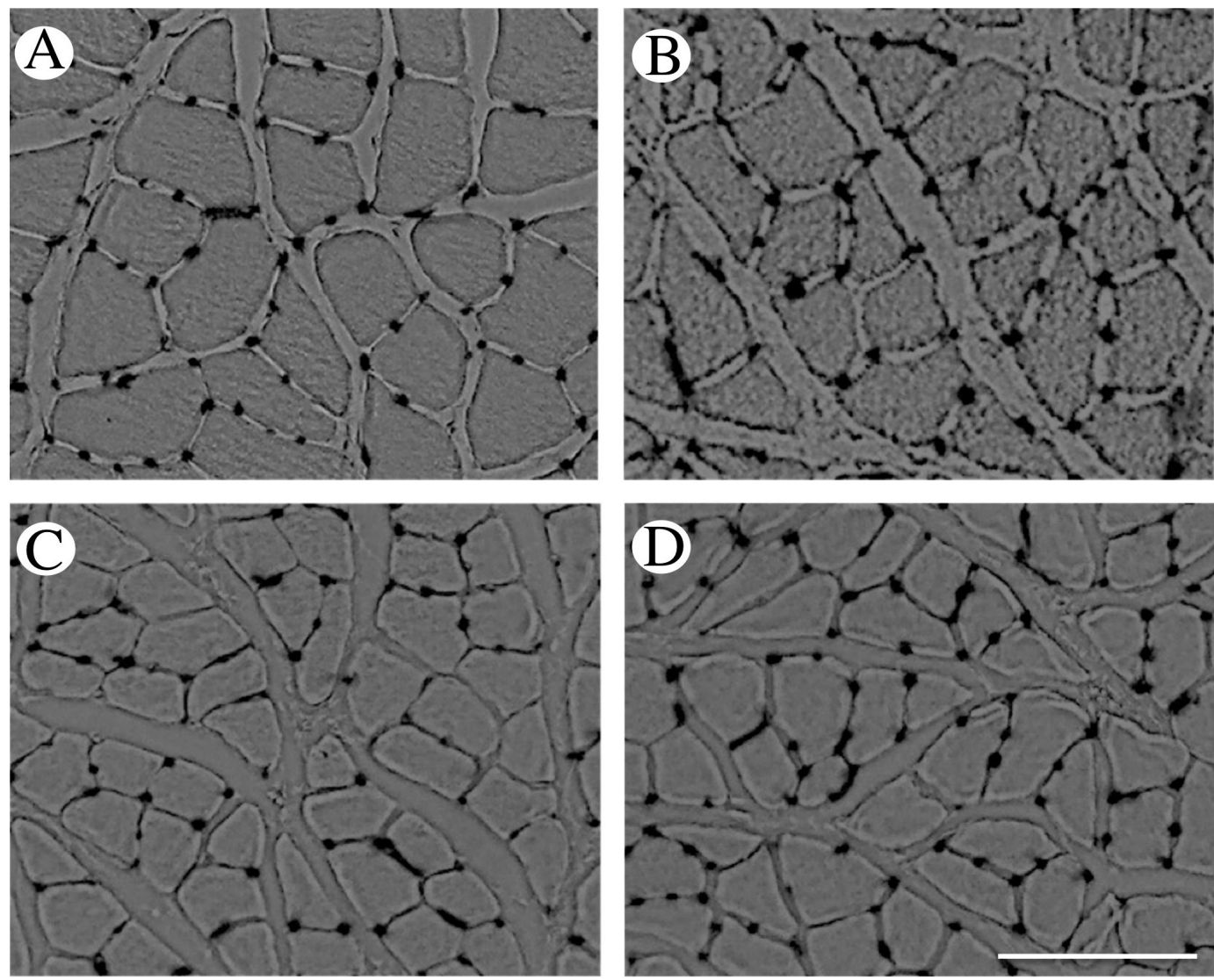

Figure 1. Transverse sections of the soleus muscles stained for alkaline phosphatase. Representative stained sections are shown for the control (A), control with L-arginine supplementation (B), hindlimb unloading (C), and hindlimb unloading with L-arginine supplementation (D) groups. Capillaries are visualized as black dots. Bar $=100 \mu \mathrm{m}$.

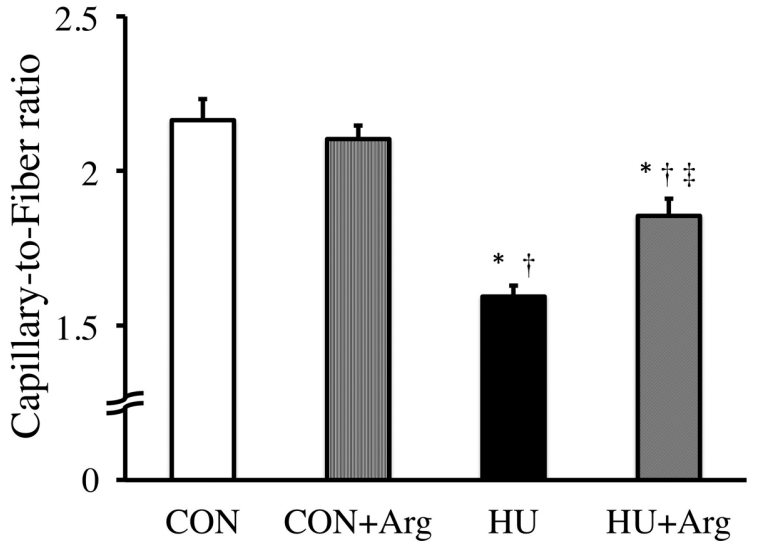

Figure 2. Capillary-to-fibre ratio in the soleus muscles. CON, control; CON+Arg, control with L-arginine supplementation; $\mathrm{HU}$, hindlimb unloading; HU+Arg, hindlimb unloading with L-arginine supplementation. Values are presented as the mean \pm SEM. ${ }^{*}, \dagger$, and $\ddagger$ indicate significant differences from CON, $\mathrm{CON}+\mathrm{Arg}$, and $\mathrm{HU}$, respectively, at $p<0.05$.

\section{Capillary-to-fibre ratio}

Figure 1 shows representative optical micrographs of transverse sections stained for alkaline phosphatase for each group. The stained capillaries are visualized as black points. There is main effect in with or without the HU treatment and is not in with or without the L-arginine treatment. Furthermore, the $\mathrm{HU}$ treatment $\times$ the $\mathrm{L}$-arginine treatment interaction is confirmed. The $\mathrm{C} / \mathrm{F}$ ratio was significantly lower in the HU group than in the CON and $\mathrm{CON}+\mathrm{Arg}$ groups (Fig. 2). The $\mathrm{C} / \mathrm{F}$ ratio in the $\mathrm{HU}+\mathrm{Arg}$ group was significantly lower than that in the CON group. However, the $\mathrm{C} / \mathrm{F}$ ratio in the $\mathrm{HU}+\mathrm{Arg}$ group was significantly higher than that in the HU group.

\section{Integrated succinate dehydrogenase activity}

The results of integrated SDH activity calculations are shown in Figure 3. Integrated SDH activity was significantly higher in the $\mathrm{CON}$ and $\mathrm{CON}+\mathrm{Arg}$ groups than in the $\mathrm{HU}$ and $\mathrm{HU}+\mathrm{Arg}$ groups. There was no significant difference 
between the $\mathrm{CON}$ and $\mathrm{CON}+\mathrm{Arg}$ groups, or between the $\mathrm{HU}$ and $\mathrm{HU}+$ Arg groups.

\section{Angiogenic factors}

There was no significant difference in the VEGF expression level among all the groups (Fig. 4A). On the other hand, there are main effects in with or without the HU treatment and the L-arginine treatment, and the HU treatment $\times$ the $\mathrm{L}$-arginine treatment interaction. The eNOS expression level was significantly lower in the HU group than in the CON and $\mathrm{CON}+$ Arg groups (Fig. 4B). In addition, the eNOS expression level was significantly higher in the HU+Arg group than in the HU group. There was no significant difference in the eNOS expression level between the HU+Arg and $\mathrm{CON}$ groups.

\section{Discussion}

The novel findings of this study were as follows: (1) Larginine supplementation attenuated capillary regression under inactivity conditions via eNOS production in skeletal muscle, and (2) L-arginine supplementation increased the level of eNOS expression, although integrated SDH activity and VEGF expression were not increased. These results indicate that the dissociation between integrated SDH activity and capillary number is a characteristic effect of angiogenesis induced by L-arginine supplementation, and that this

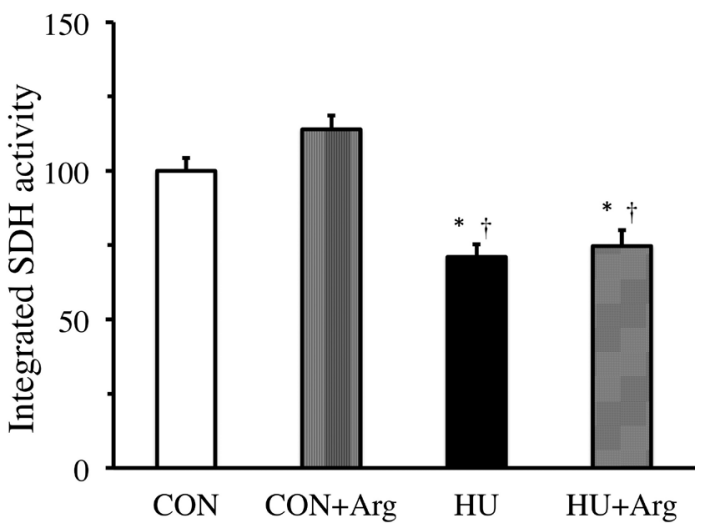

Figure 3. Integrated succinate dehydrogenase (SDH) activity in the soleus musclesValues are presented as the mean \pm SEM. ${ }^{*}$ and $\dagger$ indicate significant differences from $\mathrm{CON}$ and $\mathrm{CON}+\mathrm{Arg}$, respectively, at $p<0.05$. For abbreviations, see Fig. 2 .

intervention is an effective therapy for capillary regression in chronically unloaded muscles.

Hindlimb unloading for 7 days resulted in a decrease in wet weight, FCSA, integrated SDH activity, and $\mathrm{C} / \mathrm{F}$ ratio in the soleus muscles. It has been well established that muscle mass and size are decreased in inactivity-induced muscle atrophy (Bodine and Bacher 2014). In addition, capillary number and integrated SDH activity are reduced in atrophied muscle. The decrease in oxygen demand resulted in capillary regression in tissue (Egginton 2010). The oxygen
A

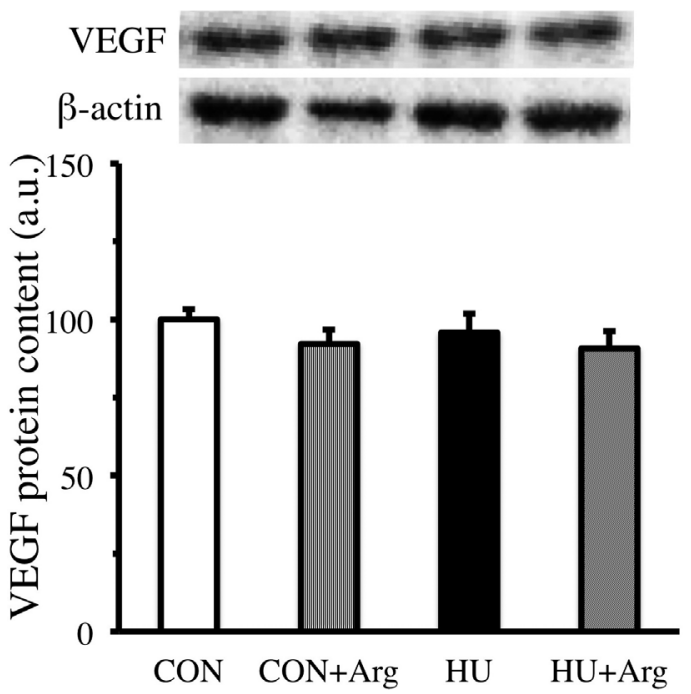

B

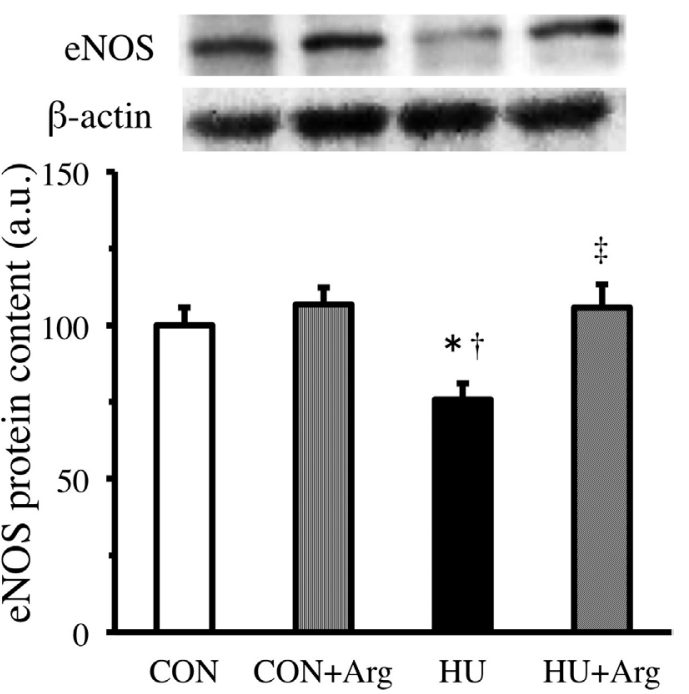

Figure 4. Expression of VEGF (A) and eNOS (B) in the soleus muscles. Representative Western blots are shown, and the histograms show quantification of the band densities (a.u., absorbance units). Values were calculated as the percentage change relative to CON and are presented as the mean \pm SEM. ${ }^{*}, \dagger$, and $\neq$ indicate significant differences from CON, CON+Arg, and HU, respectively, at $p<0.05$. For abbreviations, see Fig.2. 
demand is correlated with mitochondrial oxidative enzyme activity (Blomstrand et al. 2011), and a decrease in the oxidative enzyme activity caused by muscle disuse is also established (Fell et al. 1985). SDH activity is one of the most general mitochondrial oxidative enzyme activities. Therefore, regressed capillary number has been observed as an adaptive change to the decreased integrated SDH activity in the soleus muscles caused by hindlimb unloading.

Schrage et al. (2000) reported that decreased eNOS expression was induced by loss of physical activity. In the present study, decreased eNOS protein expression was also observed under conditions of physical inactivity. In addition, production of endothelial NO by the eNOS enzyme depends on the level of physical activity (Suvorava et al. 2004). Therefore, NO production would be decreased under inactivity conditions. Accordingly, decreased eNOS expression would induce a decrease in capillary number as an adaptation to muscular inactivity.

VEGF contributes to angiogenesis in skeletal muscle (Hoeben et al. 2004). Disappointingly, VEGF expression in the HU group was unchanged from that in the CON group in the present study. This result seemed to contradict our hypothesis. Navasiolava et al. (2010) found that the VEGF expression level was lowest after 3 days of physical inactivity; thereafter, the VEGF expression level began to recover from the decline and return to baseline. Several studies have shown that the VEGF expression level did not change after 10-14 days of physical inactivity (Wagatsuma. 2008; Kanazashi et al. 2014). Thus, in the present study, VEGF expression might have decreased and already recovered to the control level after 7 days of unloading.

L-arginine supplementation during hindlimb unloading maintained the protein level of eNOS and attenuated capillary regression in the present study. Lomonosova et al. (2011) found that L-arginine supplementation during hindlimb unloading attenuated the decrease in NO concentration of the soleus muscles. In addition, Zhang et al. (2006) reported that L-arginine supplementation led to higher eNOS expression within the pulmonary endothelium coincident with elevated NO production. Therefore, eNOS expression might attenuate capillary regression through increasing NO production in the present study. On the other hand, L-arginine could not increase the VEGF expression level, regardless of unloading. VEGF is located upstream of eNOS/NO in this angiogenic pathway. Kon et al. (2003) showed that angiogenesis was accompanied by NO production without VEGF expression in a NOS inhibitor model. Therefore, L-arginine would only activate factors downstream of eNOS in hindlimb unloading but could attenuate capillary regression in the present study.

Interestingly, capillary regression was attenuated without increasing integrated SDH activity in the present study. Several reports have shown that nutrient supplementation can prevent a decrease of mitochondrial oxidative enzyme activity and capillary regression (Kanazashi et al. 2014; Kanazawa et al. 2014). Kanazashi et al. (2014) found that astaxanthin supplementation could prevent the regression of the three-dimensional capillary network and reduction of mitochondrial oxidative enzyme activity of skeletal muscle under hindlimb unloading. In addition, Kanazawa et al. (2014) reported that nucleoprotein supplementation could prevent the decreasing of SDH activity and capillary number of skeletal muscle under hindlimb unloading. Both studies showed the correlative change between mitochondrial oxidative enzyme activity and capillary number in skeletal muscle. On the other hand, Williams et al. (2006) reported a mismatch between mitochondrial function and capillary number. Prazosin treatment resulted in an increase of capillary number in skeletal muscle, although mitochondrial volume density was unchanged. Moreover, prazosin treatment induced angiogenesis via eNOS expression (Baum et al. 2004). Thus, the dissociation between integrated SDH activity and capillary number is a characteristic effect of angiogenesis induced by L-arginine supplementation.

The expression of eNOS is primarily found in endothelial cells of vessels and microvessels (Frandsen et al. 1996). However, eNOS exists not only in endothelial cell but also in mitochondria of skeletal muscle fibres as mtNOS (Bates et al. 1996) and Kobzik et al. (1995) reported that eNOS expression in skeletal muscle fiber correlates with mitochondrial content. In the present study, the source of the eNOS would be both locations because soleus muscle had homogenized without distinction. Therefore, locality of eNOS and effect of eNOS to mitochondrial function have not been investigated.

In conclusion, L-arginine supplementation during hindlimb unloading is effective in attenuating capillary regression through maintaining the eNOS expression level. Exercise is commonly used as a countermeasure to muscle atrophy and to maintain capillary number. However, patients cannot exercise if they are restricted by disease, e.g. all diseases that are accompanied by lack of consciousness, sepsis, or injury. L-arginine supplementation may be a beneficial intervention in patients who suffer from malnutrition, because Larginine supplementation affected capillary number without increasing oxidative demand and metabolism in skeletal muscle. Therefore, these results indicate the efficiency of the L-arginine supplementation strategy during inactivity for clinical rehabilitation.

Acknowledgment. This study was supported by Grants-in-Aid for Scientific Research from the Japanese Ministry of Education, Culture, Sports, Science and Technology.

Ethical approval. All applicable international, national, and/or institutional guidelines for the care and use of animals were followed. The authors declare that they have no conflict of interest. 


\section{References}

Adrian L., Prem G., Victor S., Jean K., Ernest S., Harlan E. (1988) Calf muscle area and strength changes after five weeks of horizontal bed rest. Am. J. Sports Med. 16, 624-629 http://dx.doi.org/10.1177/036354658801600612

Bodine S. C., Bacher L. M. (2014): Skeletal muscle atrophy and the E3 ubiquitin ligases MuRF1 and MAFbx/atrogin-1. Am. J. Physiol. Endocrinol. Metab. 307, 469-484 http://dx.doi.org/10.1152/ajpendo.00204.2014

Bates T. E., Loesch A., Burnstock G., Clark J. B. (1996): Mitochondrial nitric oxide synthase: A ubiquitous regulator of oxidative phosphorylation? Biochem. Biophys. Res. Commun. 218, $40-44$ http://dx.doi.org/10.1006/bbrc.1996.0008

Baum O., Silva-Azevedo L. D., Willerding G., Wockel A., Planitzer G., Gossrau R., Pries A. R., Zakrzewicz A. (2004): Endothelial NOS is main mediator for shear stress-dependent angiogenesis in skeletal muscle after prazosin administration. Am. J. Physiol. Heart Circ. Physiol. 287, 2300-2308 http://dx.doi.org/10.1152/ajpheart.00065.2004

Bekedam M. A., van Beek-Harmsen B. J., Boonstra A., van Mechelen W., Visser F. C., van der Laarse W. J. (2003): Maximum rate of oxygen consumption related to succinatede hydrogenase activity in skeletal muscle fibres of chronicheart failure patients and controls. Clin. Physiol. Funct. Imaging 23, 337-343 http://dx.doi.org/10.1046/j.1475-0961.2003.00517.x

Blomstrand E., Krustrup P., Søndergaard H., Rådegran G., Calbet J. A., Saltin B. (2011): Exercise training induces similar elevations in the activity of oxoglutarate dehydrogenase and peak oxygen uptake in the human quadriceps muscle. Pflügers Arch. 462, 257-265 http://dx.doi.org/10.1007/s00424-011-0978-6

Booth F. W. (1982): Effect of limb immobilization on skeletal muscle. J. Appl. Physiol. 52, 1113-1118

Chalmers G. R., Roy R. R., Edgerton V. R. (1991): Motoneuron and muscle fiber succinate dehydrogenase activity in control and overloaded plantaris. J. Appl. Physiol. 71, 1589-1592

Desplanches D., Mayet M. H., Sempore B., Flandrois R. (1987): Structural and functional responses to prolonged hindlimb suspension in rat muscle. J. Appl. Physiol. 63, 558-563

Dow D. E., Cederna P. S., Hassett C. A., Kostrominova T. Y., Faulkner A. J., Dennis R. G. (2004): Number of contractions to maintain mass and force of a denervated rat muscle. Muscle Nerve 30, 77-86 http://dx.doi.org/10.1002/mus.20054

Egginton S. (2010): Muscle capillary supply takes the load. J. Physiol. 588, 4607-4608 http://dx.doi.org/10.1113/jphysiol.2010.200378

Egginton S. (2011): Physiological factors influencing capillary growth. Acta Physiol. 202, 225-239 http://dx.doi.org/10.1111/j.1748-1716.2010.02194.x

El-Hattab A. W., Emrick L. T., Craigen W. J., Scaglia F. (2012): Citrulline and arginine utility in treating nitric oxide deficiency in mitochondrial disorders. Mol. Genet. Metab. 107, 247-252 http://dx.doi.org/10.1016/j.ymgme.2012.06.018

Fell R. D., Steffen J. M., Musacchia X. J. (1985): Effect of hypokinesia-hypodynamia on rat muscle oxidative capacity and glucose uptake. Am. J. Physiol. 249, 308-312
Forstermann U., Sessa W. C. (2012): Nitric oxide synthases: regulation and function. Eur. Heart J. 33, 829-837 http://dx.doi.org/10.1093/eurheartj/ehr304

Frandsen U., Lopez-Figueroa M., Hellsten Y. (1996): Localization of nitric oxide synthase in human skeletal muscle. Biochem. Biophys. Res. Commun. 227, 88-93

http://dx.doi.org/10.1006/bbrc.1996.1472

Fujino H., Kohzuki H., Takeda I., Kiyooka T., Miyasaka T., Mohri S., Shimizu J., Kajiya F. (2005): Regression of capillary network in atrophied soleus muscle induced by hindlimb unweighting. J. Appl. Physiol. 98, 1407-1413 http://dx.doi.org/10.1152/japplphysiol.00961.2004

Hansen-Smith F. M., Blackwell L. H., Joswiak G. R. (1992): Expression of muscle capillary alkaline phosphatase is affected by hypoxia. J. Appl. Physiol. 73, 776-780

Hoeben A., Landuyt B., Highley M. S., Wildier H., Oosterom A. T. V., De Bruijn E. A. (2004): Vascular endothelial growth factor and angiogenesis. Pharmacol. Rev. 56, 549-580 http://dx.doi.org/10.1124/pr.56.4.3

Javanmard S. H., Nematbakhsh M., Mahmoodi F., Mohajeri M. R. (2009): L-Arginine supplementation enhances eNOS expression in experimental model of hypercholesterolemic rabbits aorta. Pathophysiology 16, 9-13 http://dx.doi.org/10.1016/j.pathophys.2008.11.003

Kanazashi M., Tanaka M., Murakami S., Kondo H., Nagatomo F., Ishihara A., Roy R. R., Fujino H. (2014): Amelioration of capillary regression and atrophy of the soleus muscle in hindlimbunloaded rats by astaxanthin supplementation and intermittent loading. Exp. Physiol. 99, 1065-1077 http://dx.doi.org/10.1113/expphysiol.2014.079988

Kanazawa Y., Maekawa K., Okumura Y., Fujita N., Fujino H. (2014): Preventive effect of nucleoprotein on hindlimb unloadinginduced capillary regression in rat soleus muscle. Biotech. Histochem. 89, 220-227 http://dx.doi.org/10.3109/10520295.2013.835444

Kaneguchi A., Ozawa J., Kawamata S., Kurose T., Yamaoka K. (2014): Intermittent whole-body vibration attenuates a reduction in the number of the capillaries in unloaded rat skeletal muscle. BMC Musculoskel. Disord. 15, 315 http://dx.doi.org/10.1186/1471-2474-15-315

Kim S. J., Roy R. R., Kim J. A., Zhong H., Haddad F., Baldwin K. M., Edgerton V. R. (2008): Gene expression during inactivity-induced muscle atrophy: effects of brief bouts of a forceful contraction countermeasure. J. Appl. Physiol. 105, $1246-1254$ http://dx.doi.org/10.1152/japplphysiol.90668.2008

Kimura H., Esumi H. (2003): Reciprocal regulation between nitric oxide and vascular endothelial growth factor in angiogenesis. Acta Biochim. Pol. 50, 49-59

Kobzik L., Stringer B., Balligand J. L., Reid M. B., Stamler J. S. (1995): Endothelial type nitric oxide synthase in skeletal muscle fibers: Mitochondrial relationships. Biochem. Biophys. Res. Commun. 211, 375-381 http://dx.doi.org/10.1006/bbrc.1995.1824

Kon K., Fujii S., Kosaka H., Fujiwara T. (2003): Nitric oxide synthase inhibition by $\mathrm{N}(\mathrm{G})$-nitro-l-arginine methyl ester retards vascular sprouting in angiogenesis. Microvasc. Res. 65, 2-8 http://dx.doi.org/10.1016/S0026-2862(02)00011-0 
Llevadot J., Asahara T. (2002): Effects of statins on angiogenesis and vasculogenesis. Rev. Esp. Cardiol. 55, 838-844 http://dx.doi.org/10.1016/S0300-8932(02)76713-4

Lomonosova Y. N., Kalamkarov G. R., Bugrova A. E., Shevchenko T. F., Kartashkina N. L., Lysenko E. A., Shvets V. I., Nemirovskaya T. L. (2011): Protective effect of L-arginine administration on proteins of unloaded $\mathrm{m}$. soleus. Biochemistry 76, 571-580 http://dx.doi.org/10.1134/s0006297911050075

Milkiewicz M., Hudlicka O., Brown M. D., Silgram H. (2005): Nitric oxide, VEGF, and VEGFR-2: interactions inactivity-induced angiogenesis in rat skeletal muscle. Am. J. Physiol. Heart Circ. Physiol. 289, 336-343 http://dx.doi.org/10.1152/ajpheart.01105.2004

Morey E. R., Sabelman E. E., Turner R. T., Baylink D. J. (1979): A new rat model simulating some aspects of space flight. Physiologist 22, 23-24

Murohara T., Witzenbichler B., Spyridopoulos I., Asahara T., Ding B., Sullivan A., Losordo D. W., Isner J. M. (1999): Role of endothelial nitric oxide synthase in endothelial cell migration. Arterioscler. Thromb. Vasc. Biol. 19, 1156-1161 http://dx.doi.org/10.1161/01.ATV.19.5.1156

Nachlas M. M., Tsou K. C., De Souza E, Cheng C. S., Seligman A. M. (1957): Cytochemical demonstration of succinic dehydrogenase by the use of a new p-nitrophenyl substituted ditetrazole. J. Histochem. Cytochem. 5, 420-436 http://dx.doi.org/10.1177/5.4.420

Navasiolava N. M., Dignat-George F., Sabatier F., Larina I. M., Demiot C., Fortrat J. O., Gauquelin-Koch G., Kozlovskaya I. B., Custaud M. A. (2010): Enforced physical inactivity increases endothelial microparticle levels in healthy volunteers. Am. J. Physiol. Heart Circ. Physiol. 299, 248-256 http://dx.doi.org/10.1152/ajpheart.00152.2010

Pachter B. R., Eberstein A. (1984): Neuromuscular plasticity following limb immobilization. J. Neurocytol. 13, 1013-1025 http://dx.doi.org/10.1007/BF01148599

Roudier E., Gineste C., Wazna A., Dehghan K., Desplanches D., Birot O. (2010): Angio-adaptation in unloaded skeletal muscle: new insights into an early and muscle type-specific dynamic process. J. Physiol. 588, 4579-4591 http://dx.doi.org/10.1113/jphysiol.2010.193243

Roy R. R., Zhong H., Hodgson J. A., Grossman E. J., Siengthai B., Taimadge J., Edgerton V. R. (2002): Influences of electromechanical events in defining skeletal muscle properties. Muscle Nerve 26, 238-251 http://dx.doi.org/10.1002/mus.10189
Russo T. L., Peviani S. M., Freria C. M., Gigo-Benato D., Geuna S., Salvani T. F. (2007): Electrical stimulation based on chronaxie reduces atrogin- 1 and myoD gene expressions in denervated rat muscle. Muscle Nerve 35, 87-97 http://dx.doi.org/10.1002/mus.20668

Sandonà D., Desaphy J. F., Camerino G. M., Bianchini E., Ciciliot S., Danieli-Betto D., Dobrowolny G., Furlan S., Germinario E., Goto K. et al. (2012): Adaptation of mouse skeletal muscle to long-term microgravity in the MDS mission. PLoS One 7, e33232 http://dx.doi.org/10.1371/journal.pone.0033232

Schrage W. G., Woodman C. R., Laughlin M. H. (2000): Hindlimb unweighting alters endothelium-dependent vasodilation and ecNOS expression in soleus arterioles. J. Appl. Physiol. 89, 1483-1490

Suvorava T., Lauer N., Kojda G. (2004): Physical inactivity causes endothelial dysfunction in healthy young mice. J. Am. Coll. Cardiol. 15, 1320-1327 http://dx.doi.org/10.1016/j.jacc.2004.06.030

Tadaishi M., Miura S., Kai Y., Kano Y., Oishi Y., Ezaki O. (2011): Skeletal muscle-specific expression of pgc-1a-b, an exerciseresponsive isoform, increases exercise capacity and peak oxygen uptake. PLoS One 6, e28290

http://dx.doi.org/10.1371/journal.pone.0028290

Wagatsuma A. (2008): Effect of hindlimb unweighting on expression of hypoxia-inducible factor- $1 \alpha$, vascular endothelial growth factor, angiopoietin, and their receptors in mouse skeletal muscle. Physiol Res. 57, 613-620

Williams J. L., Cartland D., Hussain A., Egginton S. (2006): A differential role for nitric oxide in two forms of physiological angiogenesis in mouse. J. Physiol. 3, 445-454 http://dx.doi.org/10.1113/jphysiol.2005.095596

Zhang J., Xia L., Zhang X., Yang W., Bai M. (2006): Influence of 1arginine on the expression of eNOS and COX2 in experimental pulmonary thromboembolism. J. Huazhong Univ. Sci. Technol. Med. Sci. 26, 524-527 http://dx.doi.org/10.1007/s11596-006-0509-4

Ziche M., Morbidelli L., Choudhuri R., Zhang H. T., Donnini S., Granger H. J., Bicknell R. (1997): Nitric oxide synthase lies downstream from vascular endothelial growth factor-induced but not basic fibroblast growth factor-induced angiogenesis. J. Clin. Invest. 99, 2625-2634 http://dx.doi.org/10.1172/JCI119451

Received: January 19, 2016

Final version accepted: April 12, 2016

First published online: July 7, 2016 